Original Research Article

\title{
A study of correlation between Platelet Volume Indices (PVI) in patients of Dyslipidemia in tertiary care hospital
}

\author{
Maru A.M. ${ }^{1}$, Kokani M.J.B. ${ }^{2}$ \\ ${ }^{1}$ Dr. Alpesh M. Maru, Department of Pathology, ${ }^{2}$ Dr. Mayur Jitubhai Kokani, Department of Pathology, both authors are \\ affiliated with GMERS Medical College, Junagadh, Gujarat, India.
}

Corresponding Author: Dr. Mayur Jitubhai Kokani, Department of Pathology, GMERS Medical College, Junagadh, Gujarat, India. E-mail- mkokon11@gmail.com

\begin{abstract}
Background: The dyslipidemia is a risk factors for cardiovascular disease. Ischemic heart disease is the leading cause of death world wide. Platelet volume reflects platelet reactivity and has been suggested as an independent risk factor for ischemic events in cardiovascular disease. We have shown preliminary results on production of larger platelets by Mean Platelets Volume (MPV) and Platelets Distribution Width (PDW) determination in a group of patients with lipid profile abnormalities. Materials \& Method: The study was performed in patients coming to GMERS Medical College, Junagadh from June 2016 to December 2017. Blood sample was collected in plain tube for lipid profile analysis, EDTA vaccutainer for haematological analysis. PVI were obtained by using Horriba 5 part auto mated cell counter \& Lipid profile parameters by fully automated analyzer Roche's COBAS INTEGRA 400plus. Results: our study comprising of 500 patient's samples and 50 control samples, In patient's group high levels of total cholesterol correlates more with high value of PDW than with MPV. Conclusion: PVI like MPV and PDW are strongly associated with dyslipidemia. The estimation of these PVI can be considered as an early, economical and rapid procedure for identification of complication sin hyper lipidemic patients.
\end{abstract}

Keywords: PDW-Platelet distribution width, MPV-Mean platelet volume, PVI-platelet volume indices.

\section{Introduction}

The dyslipidemia is a risk factors for cardiovascular disease (CVD) and diabetes. These factors include elevated triglyceride levels, low high-density lipoprotein levels, elevated cholesterol and obesity [1]. Non communicable diseases which include Diabetes mellitus and cardiovascular disease are world's biggest killer diseases, estimated to cause 3.5 millions death each year. Eighty percent of them are found in the low and middle income countries.

The WHO has developed an action plan for implementation of global strategies in prevention and control of non communicable diseases. Ischemic heart disease is the leading cause of death world wide. Platelets have definite role in causing its pathogenesis and its complications. A variety of data indicate patients with dyslipidemia have a high risk of developing cardiovascular morbidity and mortality [2,3]. Platelets play an important role in the pathogenesis of thrombosis and atherosclerosis. Activated platelets interact with

Manuscript received: $28^{\text {th }}$ July 2018

Reviewed: $6^{\text {th }}$ August 2018

Author Corrected: $14^{\text {th }}$ August 2018

Accepted for Publication: $19^{\text {th }}$ August 2018 endothelium and other inflammatory cells by the action of different molecules present on the platelet surface and/or stored in platelets granules, as P-selectin [4]. Platelet volume reflects platelet reactivity ${ }^{[5]}$ and has been suggested as an independent risk factor for ischemic events in cardiovascular disease [6]. Large platelets are metabolically and enzymatically more active than small platelets and produce more thromboxane A2 [7, 8]. Individuals with dyslipidemia have more tendencies to form atherosclerosis plaques with a consequent increasing consumption of platelets. We have shown preliminary results on production of larger platelets by MPV and PDW determination in a group of patients with lipid profile abnormalities [9].

The objective of the study was to study platelet parameters in the spectrum of dyslipidemia.

\section{Materials and methods}

A Prospective study was performed in patients coming to GMERS Medical College, Junagadh from June 2016 to December 2017. Random Blood samples were 
collected in plain tube for lipid profile analysis, EDTA (Ethy lene diaminetetraaceticacid) vaccutainer for haematological analysis under standard aseptic procedure.

Inclusion Criteria's: All routine patients of all age group

\section{Exclusion Criteria's}

1. Child below 5 years.

2. Patients with thrombocytopenia.

3. Patients on chemotherapy.

4. Patients received recent blood transfusion.

Sample was taken after 10-12 hours fasting so triglyceride level doesn't get affected. All patients' and controls' samples were processed within 2 hours of sample collection. All haematological parameter were processsed using Horriba 5 part auto mated cell counter.

\section{Original Research Article}

Automated cell counters, have made the plate let count (PC) and the plate let volume indices (PVI) - mean platelet volume (MPV), platelet distribution width (PDW) routinely available in most clinical laboratories.

Proper quality measures like internal (IQC) and external quality (EQAS) were done through out the study. All Biochemistry parameters have been analyzed using fully automated analyzer Roche's COBAS INTEGRA 400 plus. And HDL-C, Total Cholesterol and Triglycerides were measured by enzymatic methods.

The obtained parameters were evaluated using descriptive statistical analysis.

Statistical analyses were performed using the IBM SPSS (statistical Package for the Social Sciences v 20.0) and Microsoft Office Excel 2007 software. The p value $<0.05$ was taken as significant.

\section{Observations \& Results}

Table-1: Correlation between various parameters of lipid profile and platelet indices in Case group.

\begin{tabular}{|c|c|c|c|}
\hline \multicolumn{3}{|c|}{ Patient Data } \\
\hline Parameter & No. of Patients with high value & High PDW & High MPV \\
\hline Total Cholesterol & 500 & 499 & 449 \\
\hline Triglyceride & 247 & 246 & 221 \\
\hline HDL & 220 & 220 & 406 \\
\hline LDL & 452 & 451 & 200 \\
\hline
\end{tabular}

Table-2: Correlation between various parameters of lipid profile and platelet indices in Control group.

\begin{tabular}{|c|c|c|c|}
\hline \multicolumn{3}{|c|}{ Control Data } \\
\hline Parameter & No. of Control & High PDW & High MPV \\
\hline Total Cholesterol & 50 & 02 & 00 \\
\hline Triglyceride & 50 & 01 & 00 \\
\hline HDL & 50 & 00 & 00 \\
\hline LDL & 50 & 00 & 00 \\
\hline
\end{tabular}

In our study comprising of 500 patient's samples and 50 control samples, we have observed that in patient's group high levels of total cholesterol correlates more with high value of PDW (Platelet distribution width) than with MPV (Mean Platelet Volume). Same observation was made in case of high levels of Triglyceride, High Density lipoproteins (HDL) and Low density lipoproteins (LDL). These observations are summarized in table-1.

Out of 500 samples, we have included 180 dyslipidemic patients with uncontrolled diabetes. Here also we observed high MPV and PDW.

In control group, out of 50 samples, only 2 samples showed high values of PDW and only one sample showed high MPV. This observation is summarized in Table-2. 


\section{Original Research Article}

\section{Discussion}

Dyslipidemia, often an asymptomatic hidden state of blood might be the root behind a large number of complications including the life- threatening thromboembolic events. Role of platelets in such thromboembolic events is well-known and various platelet volume indices have been largely studied in these conditions.

Total cholesterol, triglycerides, and HDL cholesterol are measured directly. TC and TG values reflect cholesterol and TGs in all circulating lipoproteins, including chylomicrons, VLDL, intermediate-density lipoprotein (IDL), LDL, and HDL.

TC and HDL cholesterol can be measured in the non fasting state, but most patients should have all lipids measured while fasting (usually for 12 hour) for maximum accuracy and consistency. LDL cholesterol values are most often calculated as the amount of cholesterol not contained in HDL and VLDL. LDL can also be measured directly using plasma ultracentrifugation, which separates chylomicrons and VLDL fractions from HDL and LDL, and by an immunoassay method. Direct measurement may be useful in some patients with elevated TGs.

Lipid measurement should be accompanied by assessment for other cardiovascular risk factors, including diabetes, hypertension, smoking.

Pre-sent study was a humble attempt to study the changes in platelet volume indices in cases of dyslipidemia.

Studies have shown that platelet count and size might be gender and age dependent [10]. Hence wehave conducted this case control study with both age and sex matched controls to avoid any such bias in our results.

The collection of samples was done in EDTA bulbs and the samples were run between 2-6 hours after vene puncture to avoid bias due to swelling of platelets in EDTA [11].

We found that all three PVI - MPV, PDW were significantly higher ( $p$-value $<0.05$ ) in the study group than the controls, i.e. hyper-lipidemic patients had significantly higher MPV, PDW than the normolipidemic patients.

The association of increased MPV, PDW, P-LCR, and platelet count with diseases related to endothelial dysfunction such as metabolic syndrome, diabetes, coronary artery disease (CAD), and malignancy has been shown in many studies $[12,13,14,15]$. PDW is a measure of plate let anisocytosis with higher value indicating reactive platelet population [16].

A practical and reliable index of platelet activation has been tested, as measurements of plate let number and size, the tendency to form aggregates and the concentration of released substances stored inplatelet granules [17]. A larger MPV is an indicator of in vivo platelet activation and it is increased in vascular diseases as myocardial and cerebral infarction [18].

We know that larger platelets are considered to be metabolically, enzymatically and functionally more active than the smaller platelets. They contain more dense granules and hence are more potent and thrombogenic and this might be a cause for hyperlipidemia being a pre-thrombotic state. This study is correlated well with other study Khemka et al [19] and Desai KN [20].

Patel DS et al [21] have performed study of 44 patients of dyslipidemia \& metabolic syndrome. Results shows that, MPV and PDW were significantly high in patients having metabolic syndrome and dyslipidemia in case group compared to control group. The correlation between variables were significant in both groups $(p<0.0005)$. these results are correlated well with our study.

Hyperlipidemic patients who have larger platelets are more likely to have an associated disease condition and the platelet volume indices may form a basis for the prediction of these diseases in hyperlipidemic patients.

An attempt was also made to correlate these platelet volume indices with the severity of hyperlipidemia. For this, PDW and MPV values were correlated with the increasing values of total cholesterol and triglyceride levels in This might be possibly due to the more likelihood of occurrence of hyper lipidemia associated complications like thrombotic diseases with in creaseinits severity. our study and these results are comparable with some other study also like Khandekar M.M. [22], Fuchs J[23].

We have observed that that increased MPV, PDW is associated with poor glycometabolic control with dyslipidemic patients.Insulin resistance plays a pivotal role in the development of diabetic dyslipidemia by influencing several factors. similar findings are observed in study of Desai KN [24]. 


\section{Original Research Article}

\section{Conclusion}

PVI (Platelet Volume Indices) like MPV and PDW are strongly associated with dyslipidemia. The present study highlights the plate let volume indices in hyperlipidemic patients compared to control subjects. The results showed that larger platelets are present in patients with metabolic syndrome \& dyslipidemia.

Because larger platelets are more reactive, they can contribute to an increased risk for cardiovascular diseases as a complication of metabolic syndrome. So, estimation of these PVI can be considered as an early, economical and rapid procedure for identification of complications in hyper lipidemic patients.

Also, further studies will elucidate the reactivity of the larger platelets and the utility of the new parameter in assessing qualitative abnormalities in platelets.

We conclude that high MPV and PDW can be considered as biomarkers for early detection of impending diabeteic complications like micro vascular complications.

So these are very cost effective revolutionary markers for early detections of such complications in developing countries at low cost.

Funding: Nil, Conflict of interest: None initiated Permission from IRB: Yes

\section{References}

1. Ross R. The pathogenesis of atherosclerosis--an update. DOI:10.1056/NEJM198602203140806

2. Bartnik M, Malmberg K, Norhammar A, et al. Newly detected abnormal glucose tolerance: an important predictor of long-term outcome after myocardial infarction. DOI:10.1016/j.ehj.2004.09.021

3. Gorter PM, Olijhoek JK, van der Graaf Y, et al. Prevalence of the metabolic syndrome in patients with coronary heart disease, cerebrovascular disease, peripheral arterial disease or abdominal aortic aneurysm. DOI:10.1016/j.atherosclerosis.2003.12.033

4. Huo Y, Ley KF. Role of platelets in the development of atherosclerosis. Trends Cardiovasc Med. 2004 Jan; 14 (1):18-22.

5. Martin JF, Plumb J, Kilbey RS, Kishk YT. Changes in volume and density of platelets in myocardial infarction. Br Med J (Clin Res Ed). 1983 Aug 13; 287 (6390):456-9.
6. Tsiara S., Elisaf M., Jagroop I.A. \& Mikhailidis D. Platelets as predictor of vascular risk: is there a practical index of platelet activity? Clinical Applied Thrombosis/ Hemostasis 2003;9:177-90

7. Corash L, Tan H, Gralnick HR Heterogeneity of human whole blood platelet sub populations. I. Relationship between buoyant density, cell volume, and ultrastructure. Blood. 1977 Jan; 49(1):71-87

8. Thompson CB, Eaton KA, Princiotta SM, et al. Size dependent platelet subpopulations: relationship of platelet volume to ultrastructure, enzymatic activity, and function. Br J Haematol. 1982 Mar; 50 (3): 509-19.

9. Grotto HZ, Noronha JF. Platelet larger cell ratio (PLCR) in patients with dyslipidemia. DOI:10.1111/j. 1365-2257.2004.00634.x

10. Toryila JE, Amadi K, Adelaiye AB. Platelet count and mean platelet volume amongst elderly Nigerians. Science World J 2009;4:15-8

11. Thompson CB, Diaz DD, Quinn PG, et al. The role of anticoagulation in the measurement of platelet volumes. Am J Clin Pathol. 1983 Sep;80(3):327-32.

12. Aypak C, Türedi O, Bircan MA, Yüce A. Could mean platelet volume among complete blood count parameters be a surrogate marker of metabolic syndrome in pre-pubertal children? Platelets. 2014; 25: 393-98

13. Li JY, Li Y, Jiang Z, et al. Elevated mean platelet volume is associated with presence of colon cancer. Asian Pac J Cancer Prev. 2014;15(23):10501-4.

14. Li S, Zhu CG, Guo YL, et al. The relationship between the plasma PCSK9 levels and platelet indices in patients with stable coronary artery disease. doi: 10 . 5551/jat.25841. Epub 2014 Sep 2.

15. Zaccardi F, Rocca B, Pitocco D, et al. Platelet mean volume, distribution width, and count in type 2 diabetes, impaired fasting glucose, and metabolic syndrome : a meta- analysis. DOI: 10. 1002/ dmrr. 2625.

16. Ranjith MP, Divya R, Mehta VK, et al. Significance of platelet volume indices and platelet count in ischaemic heart disease. doi: 10.1136/jcp.2009. 066787. 


\section{Original Research Article}

17. Tsiara S, Elisaf M, Jagroop IA, Mikhailidis DP. Platelets as predictors of vascular risk: is there a practical index of platelet activity? v Clin Appl Thromb Hemost. 2003 Jul; 9(3): 177-90.

18. Martin JF, Plumb J, Kilbey RS, Kishk YT. Changes in volume and density of platelets in myocardial infarction. Br Med J (Clin Res Ed). 1983 Aug 13;287 (6390): 456-9.

19. Khemka R, Kulkarni K. Study of relationship between Platelet Volume Indices and Hyperlipidemia. Annals of Pathology and Laboratory Medicine. 2014; 1 (1):8-14

20. Khandekar MM, Khurana AS, Deshmukh SD, et al. Platelet volume indices in patients with coronary artery disease and acute myocardial infarction: an Indian scenario. DOI:10.1136/jcp.2004.025387
21. Patel DS, Desai KN, Gami BN, Joshi HJ, Sapre JS. Platelet Volume Indices (Pvi) In Metabolic Syndrome (Ms) Int J Pharm Bio Sci 2014;5(1):59 - 64

22. Khandekar MM, Khurana AS, Deshmukh SD, et al. Platelet volume indices in patients with coronary artery disease and acute myocardial infarction: an Indian scenario. DOI:10.1136/jcp.2004.025387

23. Fuchs J, Beigel Y, Green P, et al. Big platelets in hyperlipidemic patients. J Clin Pharmacol. 1992 Jul;32 (7): 639-42.

24. Desai KN, Lakum NR An Evaluation Study of Platelet Volume Indices (PVI) in Type-2 Diabetes Mellitus and its Micro and Macro Vascular Complications. APALM 2018;5(1):478-83

\section{How to cite this article?}

Maru A.M, Kokani M.J.B. A study of correlation between Platelet Volume Indices (PVI) in patients of Dyslipidemia in tertiary care hospital. Trop J Path Micro 2018;4(4):314-318.doi:10. 17511/ jopm. 2018.i4.03 